\title{
Influence de produits phytosanitaires (herbicides et fongicides) sur la croissance et l'infectivité de souches pures de Frankia isolées de nodules d'aulne
}

\author{
A. MOIROUD, P. SIMONET et M. FAURE-RAYNAUD \\ Laboratoire de Microbiologie physiologique et appliquée \\ Université Claude-Bernard, Lyon I \\ 43, bd du 11 Novembre 1918, F 69622 Villeurbanne Cedex
}

\begin{abstract}
Résumé
L'influence de 4 herbicides, Légurame (carbétamide), Dévrinol (napropamide), Fulton (napropamide + nitralin), Comodor (butam) et d'un fongicide, Cryptonol (oxyquinoléine) sur la croissance et l'infectivité de cultures pures de Frankia isolées de nodules d'aulne a été étudiée. Tous ces produits phytosanitaires sont couramment utilisés en traitement du sol pour lutter contre les mauvaises herbes ou les champignons phytopathogènes. A faibles concentrations les 4 herbicides utilisés n'inhibent pas la croissance de Frankia. Par contre aucun développement de ce microorganisme n'a pu être obtenu en présence de Cryptonol. De faibles concentrations en herbicide dans le milieu n'inhibent pas la formation de nodosités sur les racines de jeunes plants d'aulne. A fortes concentrations les herbicides utilisés limitent fortement le développement racinaire et aucune nodosité n'est formée. Le cryptonol n'affecte pas la croissance de la plante-hôte mais empêche la nodulation par action sur le symbiote microbien.
\end{abstract}

Mots clés : Aulne, Frankia, herbicide, fongicide, nodulation.

\section{Introduction}

L'aulne glutineux, Alnus glutinosa Gaern. est maintenant considéré comme une espèce particulièrement intéressante tant pour la production de biomasse que pour l'amélioration de la productivité d'autres ligneux associés en culture mixte (PLASS, 1977 ; Saucier, 1977 ; Kellison \& White, 1979 ; Fessenden, 1979 ; Askew \& LANE, 1979). Il s'agit en effet d'une espèce fixatrice symbiotique d'azote, capable de se développer sur des sols médiocres très déficitaires en azote combiné (Crocker \& Major, 1955 ; Dale, 1963 ; Plass, 1977 ; Molroud \& Capellano, 1978). En outre, grâce aux chutes annuelles d'une litière abondante et à forte teneur en azote (Silvester, 1977) l'aulne est capable d'enrichir rapidement le sol en cet élément (Viereck, 1966; Molroud \& Capellano, 1979). Depuis la mise au point des techniques d'isolement et de culture in vitro de l'endophyte des actinorhizes (CALlAHAM et al., 1978 ; LALONDE et al., 1981) il est possible d'inoculer avec les souches les plus performantes de Frankia de jeunes plants d'aulne qui seront ensuite mis en place sur le terrain. On a pu en effet montrer que de la souche de Frankia 
uitilisée dépend, pour une large part, l'efficience de la symbiose (Dilion \& Baker, 1982 ; Maynard \& Hall, 1980 ; Normani) \& Lalonde, 1982). Toutefois, lors du passage en pépinière ou de la mise en place définitive, les racines nouvellement formées peuvent être colonisées par les souches autochtones du sol, qui peuvent être moins efficientes que la souche pure utilisée pour l'inoculation. Il peut donc être intéressant de limiter, au moins pendant la période critique de reprise des jeunes plants, ces infections secondaires par utilisation de composés actifs sur les formes libres de Frankia et à prix de revient modéré. De même, pour favoriser la reprise sur le terrain, il peut être nécessaire de lutter contre la concurrence des mauvaises herbes souvent favorisées par la préparation du sol (MAYNARD \& Hal.l, 1980). Or, et à la différence de ce que l'on rencontre dans le cas de la symbiose Rhiz:obiumLégumineuses (GIBSON, 1977 ; GIARDINI et al., 1978), les connaissances concernant l'impact des produits phytosanitaires sur Frankia ou la symbiose actinorhizienne sont extrêmement réduites (Moiroud \& Faure-Raynaud, 1983). Il nous a donc paru intéressant d’entreprendre une étude approfondie de linfluence de certains produits phytosanitaires (herbicides et fongicides) sur le développement in vitro de souches pures de Frankia, leur infectivité en présence de tels composés dans le milieu ainsi que sur l'efficience de la symbiose. Nous rapporterons dans cette note les résultats obtenus concernant laction de 4 herbicides et d'un fongicide à large spectre sur la croissance et l'infectivité de souches pures de Frankia.

\section{Matériel et méthodes}

\subsection{Produits phytosanitaires utilisés}

\subsection{Herbicides}

Les herbicides utilisés appartiennent à deux grands groupes chimiques, celui des amides et celui des toluidines.

\subsection{Groupe des amides}

2.1111. Carbétamide $=\mathrm{C}_{12 .} \mathrm{H}_{16} \mathrm{O}_{3} \mathrm{~N}_{2 .}$ ou phénylcarbamoyloxy-2 N-éthylpropionamide, isomère D. Dénomination commerciale : Légurame. Formulation : poudre contenant 70 p. 100 de matière active.

2.1112. Napropamide $=\mathrm{C}_{17} \mathrm{H}_{21} \mathrm{NO}_{2 .}$ ou $\mathrm{N}, \mathrm{N}$-diéthylpropionamide ( $\alpha$-naphtoxy). Dénomination commerciale : Dévrinol. Formulation : poudre contenant $50 \mathrm{p}$. 100 de matière active.

2.1113. Butam $=\mathrm{C}_{15} \mathrm{H}_{233} \mathrm{NO}$ ou $\mathrm{N}$-benzyl-N-isopropyltriméthylacétamide. Désignation commerciale : Comodor. Formulation : liquide contenant 72 p. $100(\mathrm{p} / \mathrm{v})$ de matière active.

\subsection{Groupe des toluidines}

2.112.1. Napropamide + nitralin $=$ association du composé précédent et du nitralin : $\mathrm{C}_{13} \mathrm{H}_{19} \mathrm{O}_{63} \mathrm{~N}_{33} \mathrm{~S}$ ou méthylsulfonyl-4-dinitro-2,6 N,N-dipropylaniline. Désigna- 
tion commerciale : Fulton. Formulation : poudre contenant 28 p. 100 de napropamide et 32 p. 100 de nitralin.

\subsection{Fongicide}

2.121. Oxyquinoléine $=\mathrm{C}_{9} \mathrm{H}_{7} \mathrm{OH}$ ou 8-hydroxyquinoléine. Désignation commerciale : Cryptonol liquide. Formulation : liquide contenant $140 \mathrm{~g} / 1$ de matière active.

\subsection{Souches de Fankia utilisées}

Toutes les souches utilisées ont été isolées au laboratoire à partir de nodules récoltés dans la nature. Sur les 5 souches étudiées, 4 proviennent de nodules d'Alnus glutinosa (AgN21; AgN10ai; AgN12a; AgN24 IIh) et une de nodules d'Alnus viridis (Av16a).

\subsection{Influence des herbicides sur la croissance in vitro de souches pures de Frankia}

Après stérilisation par filtration, les herbicides ont été ajoutés au milieu de culture habituel (FAURE-RAYNAUD et al., 1984) de façon à obtenir les concentrations en produit commercial suivantes : $20 \mu \mathrm{tg} / \mathrm{ml} ; 50 \mu \mathrm{g} / \mathrm{ml} ; 100 \mu \mathrm{lg} / \mathrm{ml} ; 200 \mu \mathrm{g} / \mathrm{ml}$. Toutefois, par suite de la très faible solubilité du Fulton, seule la concentration de $20 \mu \mathrm{g} / \mathrm{ml}$ a été utilisée. Pour chaque herbicide et chacune des concentrations 5 tubes de milieu ont été ensemencés, chacun avec $0,5 \mathrm{ml}$ d'une suspension homogène de Frankia. Après 4 semaines de culture à $28^{\circ} \mathrm{C}$, les colonies ont été récoltées, lavées 2 fois dans de l'eau distillée puis homogénéisées par traitement aux ultra-sons (appareil Labsonic ; 10 secondes ; 80 watts) et la densité optique de la suspension homogène obtenue mesurée à $570 \mathrm{~nm}$. Pour chacune des souches utilisées, une série de 5 tubes de milieu sans herbicide a été ensemencée pour servir de témoin.

\subsection{Influence du Cryptonol (fongicide) sur la croissance in vitro de souches pures de Frankia}

Le même protocole expérimental a été suivi.

Les concentrations suivantes en fongicide ont été utilisées : $0,3 \mu \mathrm{ll} / \mathrm{ml} ; 1,5 \mu \mathrm{ll} / \mathrm{ml}$; $2,4 \mathrm{ul}^{\mathrm{l} / \mathrm{ml}} ; 3 \mathrm{ul} / \mathrm{ml}$.

\subsection{Influence des herbicides sur l'infectivité de souches pures de Frankia}

De jeunes plants d'aulne glutineux, provenant de la germination de graines ré-

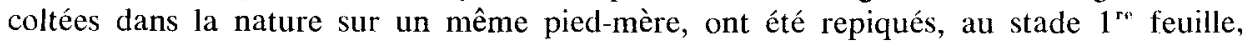
sur $15 \mathrm{~g}$ d'un substrat inerte (argile expansée) contenus dans des tubes de verre de $22 \mathrm{~mm}$ de diamètre et humidifiés avec un milieu minéral dépourvu d'azote. Chacun des herbicides utilisés a été ajouté au substrat lors du repiquage des jeunes aulnes. Pour chaque herbicide des séries de 5 tubes contenant respectivement $20 \mu \mathrm{kg}, 50 \mu \mathrm{g}$, 100 g et 200 jg de produit commercial par tube ont été utilisées. Après 3 semaines de développement en présence d'herbicide dans des conditions contrôlées ( $22^{\circ} \mathrm{C}$; 
$16 \mathrm{~h}$ de lumière ; $8 \mathrm{~h}$ d'obscurité) quelques aulnes de chaque série ont été inoculès par addition d'1 ml d'une suspension dense et homogène de la souche AgN24 IIh. Des plants d'aulne développés sur substrat totalement dépourvu d'herbicide ont été ou non inoculés avec la même souche de Frankia pour servir de témoins d'infectivité de la souche. Ils servaient également de référence pour déceler une éventuelle phytotoxicité des composés étudiés.

Après 7 semaines de développement la présence de nodules sur le système racinaire des jeunes aulnes inoculés a été recherchée.

\subsection{Influence du Cryptonol sur l'infectivité de souches pures de Frankia}

Des séries de jeunes plants d'aulne glutineux au stade $1^{\text {re }}$ feuille ont été repiqués comme précédemment sur substrat artificiel dans les conditions suivantes :

- série 1 : substrat additionné de $2 \mathrm{mg} /$ tube de $\mathrm{KNO}_{3}$,

- série 2 : substrat additionné de $2 \mathrm{mg} /$ tube de $\mathrm{KNO}_{3}$ et de $15 \times 10^{-3} \mathrm{ml} /$ tube de Cryptonol,

- série 3 : substrat additionné de $15 \times 10^{-3} \mathrm{ml} /$ tube de Cryptonol,

- série 4 : substrat seul,

— série 5 : substrat seul.

Dans les séries 1 et 2, les jeunes aulnes n'ont pas été inoculés. Dans la série 5, considérée comme témoin, l'inoculation a été réalisée par immersion et séjour pendant 1 heure du système racinaire des jeunes aulnes dans une suspension dense et homogène de Frankia. Dans la série 4, l'inoculation a été réalisée de façon comparable. Toutefois, pour cette série, 1 heure avant l'immersion des racines, $15 \times 10^{-3} \mathrm{ml}$ de Cryptonol ont été ajoutés à la suspension de Frankia. Dans la série 3, l'inoculation a été réalisée par apport, directement dans le tube de culture de l'aulne de $0,5 \mathrm{ml}$ d'une suspension dense de Frankia. Dans les séries 3, 4 et 5, le substrat artificiel était humidifié par une solution minérale dépourvue d'azote combiné. Les séries 3, 4 et 5 ont chacune été inoculées avec les souches $\operatorname{AgN} 21, \operatorname{AgN} 12 a, A g N 24$ IIh.

\section{Résultats}

\subsection{Influence des herbicides sur la croissance in vitro de souches pures de Frankia}

Les résultats obtenus sont rassemblés dans le tableau 1. On peut noter qu'aucun des herbicides utilisés n'inhibe totalement la croissance des souches pures de Frankia. Pour de faibles concentrations en Légurame ou en Dévrinol, on peut même observer une stimulation de la croissance de certaines souches (AgN21; AgN10ai; AgN12a). Par contre le Comodor réduit sensiblement la croissance in vitro de Frankia et tout particulièrement à forte concentration (tabl. 1). Ainsi lorsque le milieu de culture contient $200 \mu \mathrm{g} / \mathrm{ml}$ de Comodor, la biomasse des colonies obtenues ne représente plus que de 10 à 20 p. 100 de celle des témoins. 


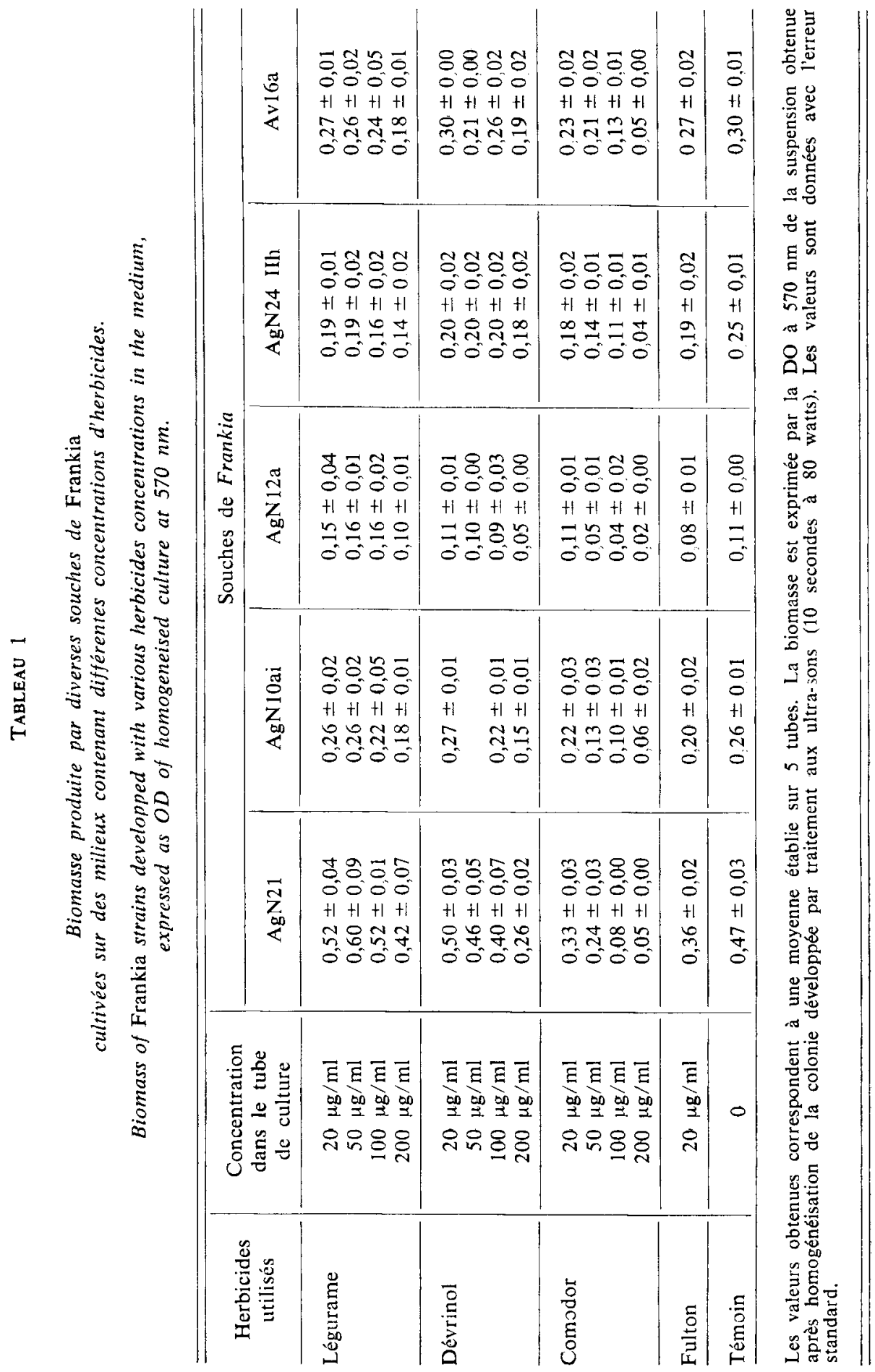




\subsection{Influence du Cryptonol sur la croissance in vitro de souches pures de Frankia}

Les résultats obtenus (tabl. 2) montrent quaucune des souches de Frankia étudiées n'est capable de se développer lorsque le milieu de culture contient du Cryptonol, même à faible concentration.

\section{TABleaU 2}

Biomasse produite par diverses souches pures de Frankia développées sur des milieux contenant différentes concentrations de Cryptonol.

Biomass of Frankia strains developped with various Cryptonol concentrations in the medium.

\begin{tabular}{|c|c|c|c|c|}
\hline \multirow{2}{*}{ Concentration en Cryptonol } & \multicolumn{4}{|c|}{ Souches de Frankia } \\
\hline & $\mathrm{AgN} 21$ & $\operatorname{AgN} 12 a$ & AgN24 Ilh & $A v N 16 a$ \\
\hline $0,3 \times 10^{--3} \mathrm{ml} / \mathrm{ml}$ & 0 & 0 & 0 & 0 \\
\hline $1,5 \times 10^{-3} \mathrm{ml} / \mathrm{ml}$ & 0 & 0 & 0 & 0 \\
\hline $2,4 \times 10^{-3} \mathrm{ml} / \mathrm{ml}$ & 0 & 0 & 0 & 0 \\
\hline $3 \times 10^{-3} \mathrm{ml} / \mathrm{ml}$ & 0 & 0 & 0 & 0 \\
\hline 0 - Témoin & $0,36 \pm 0,06$ & $0,31 \pm 0,00$ & $0.25 \pm 0,03$ & $077 \pm 0,07$ \\
\hline
\end{tabular}

Les valeurs obtenues correspondent à des moyennes ćtablies sur 4 tubes.

\section{TABleau 3}

Influence de la concentration en herbicide du substrat de culture sur la survie et la nodulation de jeunes aulnes glutineux inoculés avec une souche pure de Frankia.

Herbicide concentrations effects on nodulation of Alnus glutinosa seedlings inoculated with Frankia strains.

\begin{tabular}{c|c|c|c|c}
\hline $\begin{array}{c}\text { Concentration } \\
\text { en herbicide } \\
\text { (ug/tube) }\end{array}$ & $\begin{array}{c}\text { Nombre } \\
\text { de plants traités }\end{array}$ & $\begin{array}{c}\text { Nomóre total } \\
\text { de plants morts }\end{array}$ & $\begin{array}{c}\text { Nombre de plants } \\
\text { inozulés }\end{array}$ & $\begin{array}{c}\text { Nombre de } \\
\text { plants nodulés }\end{array}$ \\
\hline 0 & 0 & 0 & 5 & 5 \\
20 & 14 & 2 & 10 & 7 \\
50 & 19 & 7 & 12 & 8 \\
100 & 18 & 8 & 10 & 0 \\
200 & 19 & 9 & 9 & 0 \\
\hline
\end{tabular}

Lcs plants ont été inoculés 3 semaines après l'apport d'herbicide dans le substrat de culture. La présence de nodules a été recherchée 7 semaines après l'inoculation.

I. cs résultats rassemblés dans ce tableau correspondent à la sommation des résultats obtenus pour chacun des herbicides étudiés. 


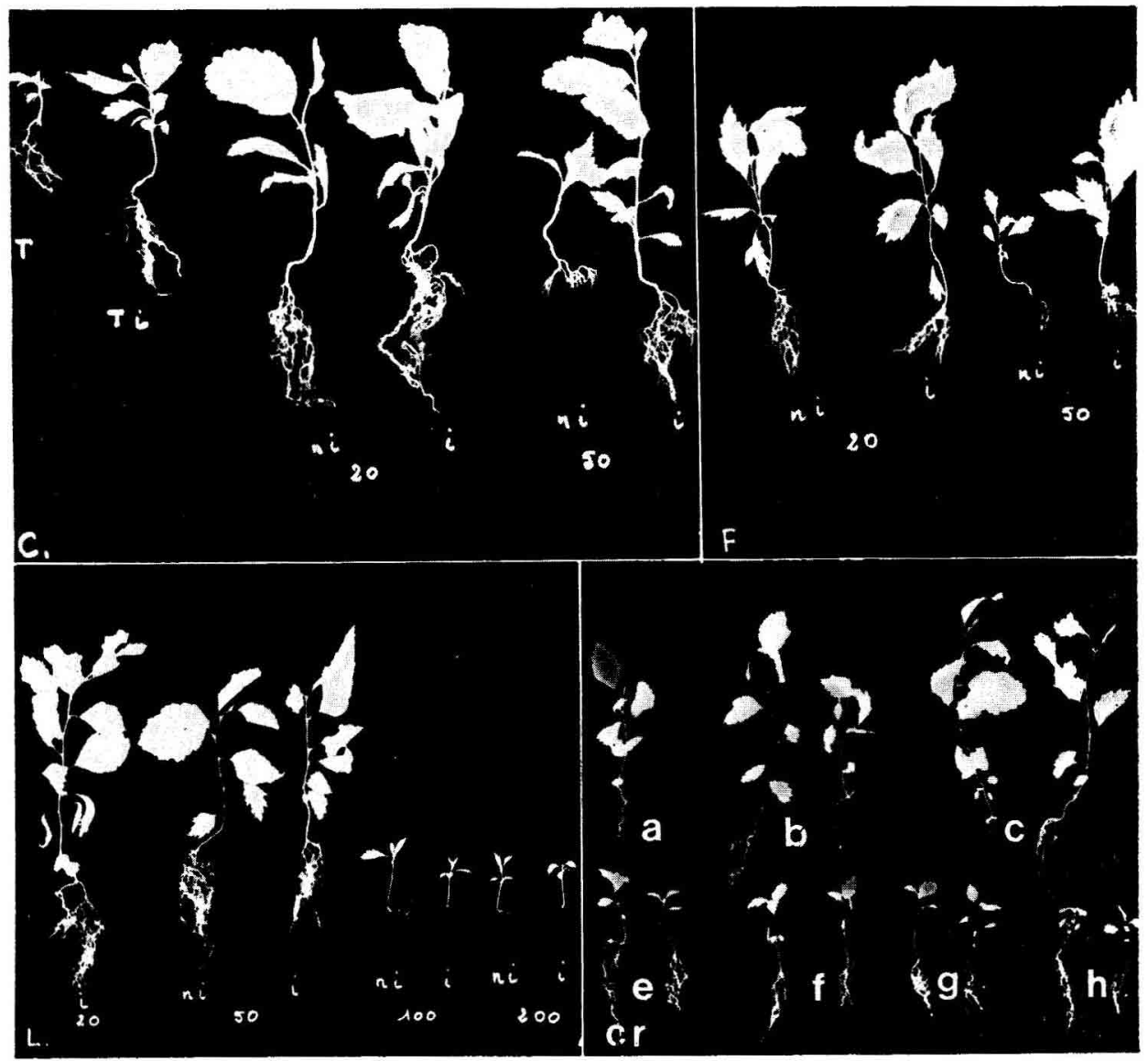

FIG. 1

Influence de différentes concentrations en herbicides ou fongicide sur la nodulation de jeunes plants d'aulne.

Influence of different concentrations of herbicides or fungicide on Alnus seedlings nodulation.

Herbicides : $\mathrm{C}=$ Comodor, $\mathrm{F}=$ Fulton, $\mathrm{L}=$ Légurame.

$\mathrm{T}=$ Témoin non inoculé sans herbicide, $\mathrm{Ti}=$ Témoin inoculé, $\mathrm{ni}=$ non inoculé, $\mathrm{i}=$ inoculé.

$20 ; 50 ; 100 ; 200=$ Concentrations on $\mu \mathrm{g} /$ tube d'herbicide.

Fongicide : $\mathrm{Cr}=$ Cryptonoi.

$\mathrm{a}=$ plant développé en présence de $\mathrm{NO}:$, $\mathrm{b}=$ plani développé en présence de $\mathrm{NO}$ : et de Cryptonol, $\mathrm{c}=$ plant développé sans $\mathrm{NO}_{3}$, et sans Cryptonol mais inoculé, e, $\mathbf{f}, g, \quad h=$ plants développés après inoculation par des souches différentes de Frankia ayant préalablement subi l'action du Cryptonol pendant $1 \mathrm{~h}$.

Herbicides : $S=$ Comodor, $F=$ Fulton, $L=$ Légurame.

$T=$ Temoin, $T i=$ inoculated temoin, $n i=$ non inoculated, $i=$ inoculated.

$20 ; 50 ; 100 ; 200=$ Concentrations of herbicides in $\mu \mathrm{g} /$ tube.

Fungicide : $\mathrm{Cr}=$ Cryptonol.

$a=$ seedling developped with $N O, b=$ seedling with NO: and Cryptonol, $c=$ seedling inoculated, without $\mathrm{NO}_{3}$ or Cryptonol, $e, f, g, h=$ seedlings inoculated with 4 different Frankia strains incubated with Cryptonol in the medium. 


\subsection{Influence des herbicides sur l'infectivité de souches pures de Frankia}

Lorsque la plante-hôte se développe en présence de 20 ltg d'herbicide la nodulation n'est pas inhibće (tabl. 3). Pour cette concentration en herbicide la croissance des plants nodulés ou non nodulés ne diffère pas sensiblement (photos 1 et 2). On remarque également que la taille des plants cultivés en présence d'herbicide est supérieure à celle des témoins développés en l'absence de tels composés.

La nodulation n'est pas non plus totalement inhibée par la présence de 50 lg d'herbicide dans le milieu de culture (tabl. 3). Cependant, pour une telle concentration, on peut observer une différence marquée entre la taille des plants inoculés et non inoculés. Ainsi en présence de Dévrinol, Comodor et Fulton, l'appareil végétatif des plants non nodulés est beaucoup moins développé que celui des plants nodulés (photos 1 et 2 ). Par contre la taille de ces jeunes aulnes non nodulés est supérieure à celle des témoins non nodulés cultivés en l'absence d'herbicide.

Lorsque le milieu contient 100 ou 200 /g d'herbicide la nodulation est totalement inhibée. La croissance des aulnes est aussi fortement affectée, l'appareil aérien est très réduit et le système racinaire ne se développe pas (photo 3).

On remarque également qu'en présence d'herbicide la mortalité des aulnes est toujours supérieure à celle observée chez les plants dévloppés en l'absence de tels composés (tabl. 3).

\subsection{Influence du Cryptonol sur l'infectivité de souches pures de Frankia}

Les systèmes racinaires de jeunes plants d'aulne inoculés avec des cultures pures de Frankia préalablement traitées par une solution de Cryptonol ne portent jamais de nodosités, même après 3 mois de culture. Les jeunes plants d'aulne ont du reste une taille réduite $(3$ à $4 \mathrm{~cm}$ ), nettement inférieure à celle des témoins inoculés $(6,5 \mathrm{~cm})$ ou des plants bénéficiant d'une alimentation nitrique $(5-6 \mathrm{~cm})$ (photo 4 ). Les feuilles, de couleur jaunâtre, témoignent d'une forte carence en azote. L'action inhibitrice du Cryptonol se manifeste également lorsque ce composé est ajouté au substrat de culture au moment de l'inoculation. Aucun des plants ainsi traités ne portait de nodosités même après 3 mois de culture. Le cryptonol ne présente, par contre, aucune action phytotoxique sur l'aulne; la taille et la biomasse des plants à nutrition nitrique en présence ou absence de fongicide ne montrent aucune différence marquée (photo 4).

\section{Discussion}

Les 4 herbicides utilisés sont actifs contre les Graminées et certaines Dicotylédones adventices. Ils ont été choisis parce qu'ils sont applicables en traitement du sol avec incorporation dans les premiers centimètres (Acta, 1982). Ce sont, en outre, des produits stables, faisant preuve d'une longue persistance (au moins 2 mois) dans le sol et à très faible toxicité (DL $50>5000 \mathrm{mg} / \mathrm{kg}$ ). Le cryptonol est aussi utilisable en désinfection du sol pour lutter contre de nombreux champignons phytopathogènes (Acta, 1982). 
Les résultats obtenus montrent que les cultures pures de Frankia sont capables de résister aux herbicides étudiés, même employés à fortes concentrations. Une telle tolérance à l'égard de ce type de composés avait déjà été signalée pour d'autres organismes fixateurs (GIARdini et al., 1978 ; VinCENT, 1977). Pour de faibles concentrations en Dévrinol et Légurame, on observe même une certaine stimulation de la croissance de Frankia. Cette stimulation pourrait être due à l'utilisation des composés accompagnant le produit actif dans la formulation commerciale car les cultures pures de Frankia sont incapables de métaboliser ou de dégrader la molécule active (Molroud \& Faure-RaYnaud, 1983).

Contrairement aux herbicides utilisés, le Cryptonol inhibe totalement la croissance des cultures pures de Frankia. Il se comporte donc aussi comme un bactéricide très efficace. Il est du reste connu que bon nombre de produits actifs contre les champignons phytopathogènes le sont aussi contre certaines bactéries, notamment celles du genre Rhizobium (FISHER et al., 1978; FiSHER et al., 1979; Fisher \& HAYES, 1981, 1982).

Les composés phytosanitaires utilisés paraissent avoir une action sur l'établissement de la symbiose. Si, pour de faibles concentrations dans le milieu, les herbicides employés n'inhibent pas la formation de nodosités, leur action sur la plante n'en est pas moins déjà manifeste. En effet la mortalité des jeunes aulnes développés en présence d'herbicide est supérieure à celle des témoins poussant en l'absence de tels composés. En outre, en présence d'herbicide, la taille des plants non inoculés est supérieure à celle des témoins; ils ne présentent pas non plus de signes extérieurs de carence en azote.

Lorsque la concentration en herbicide dans le milieu s'élève, on observe une forte réduction du système racinaire. Cette inhibition de la croissance des racines correspond du reste au mode d'action des herbicides utilisés (Acta, 1982). Dans ces conditions l'ensemble des processus de reconnaissance hôte-endophyte et d'infection, indispensables à la formation des nodules (BECKiNG, 1975 ; TORREY, 1976), ne pourraient se dérouler normalement. Dès lors il apparaît très difficile de savoir si l'absence de nodulation observée en présence de fortes concentrations en herbicide est réellement due à la perte du pouvoir infectif des souches de Frankia.

Le fongicide employé ne paraît, par contre, avoir aucune action phytotoxique sur la plante-hôte. Ce fait est à souligner car de nombreux composés de ce type diminuent l'intensité de la fixation symbiotique d'azote en affectant le développement de la plante-hôte (Fisher \& HAYEs, 1981). Ainsi, l'absence de nodulation en présence de Cryptonol est due à son action sur le symbiote bactérien. Une réduction significative du nombre de nodules formés en présence de certains fongicides a aussi été signalée dans le système Rhizobium-Trifolium (FISHER \& HAYES, 1981, 1982).

Avant de pouvoir utiliser de façon intensive des composés phytosanitaires pour favoriser le développement au champ de l'aulne de nombreuses études sont encore nécessaires, concernant notamment l'influence de ces composés sur l'établissement et l'efficience de la symbiose. De telles études devraient également être élargies à d'autres familles d'herbicides.

\author{
Reçu le 19 mars 1984.
}

Accepté le 27 octobre 1984.

\title{
Remerciements
}

Les auteurs remercient $\mathbf{M}^{\mathrm{me}} \mathbf{N}$. Guillaumaud et $\mathbf{M}^{\mathrm{He}}$ M.A. Bonnefoy pour leur aide. 


\section{Summary}

Influence of four herbicides and one fungicide on Frankia strains grown in vitro, on nodule formation and on Alder seedlings growth

Effects of four herbicides, Legurame (carbetamide), Devrinol (napropamide), Fulton (napropamide + nitralin), Comodor (butam) and one fungicide, Cryptonol (oxyquinoleine), on Frankia strains grown in vitro were studied. All these compounds are widely used on soil treatment against weeds or pathogens. At low concentrations, herbicides had no significant effects on the growth of Frankia strains but Cryptonol was highly toxic. Herbicides did not affect nodule formation at low concentrations. When high concentrations were used, growth of Alnus seedlings was restricted and no nodule lormed. Cryptonol had no effect on Alnus seedling growth but inhibited nodule formation.

\section{Références bibliographiques}

A.C.T.A., 1982. Index phytosmitaire. Ed. Acta, Paris, 585 p.

Askew J.L., LaNu C.L., 1979. Nitrogen fixing capabilities for Myrica cerifera, Llaeagnus pungens and various Alnus species grown on Piedmont sites in South Carolina. In : Symbiotic nitrogen fixation in the management of temperate forests. Eds. GORDon J.C., Wheeler C.T., Perry D.A., Corvallis Univ., p. 470.

BLCKING J., 1975. Root nodules in non-Legumes. In : The development and function of root. Eds. Torrey J., Clarkson D., Academic Press, 507-566.

Catlaham D., Dhe Tredic P., Torrey J., 1978. Isolation and cultivation in vitro of the Actinomycetes causing root nodulation in Comptonia. Science, 199, 899-902.

Crockir R.L., Major J., 1955. Soil development in relation to vegetation and surface age at Glacier Bay, Alaska, 79. J. Ecol., 43, 427-448.

DALE M.E., 1963. Interplant alder to increase growth in strip mine plantations. U.S.D.A. For. Serv. Res. Note C.S. 14, Central States For. Expt. Stat. Colombus Ohio, 4 p.

Dilion J., BAKer D., 1982. Variations in nitrogenase activity among pure-cultured Frankia strains tested on an actinorhizal plants as an indication of symbiotic compatibility. New: Phytol., e2, 215-219.

Faure-Raynaud M., Bonnefoy M.A., Perradin Y., Simonet P., Molroud A., 1984. Protoplasts formation from Frankia strains. Microbios (Sous presse).

FusSENDEN R.J., 1979. Use of actinorhizal plants for land reclamation and amenity planting in the U.S.A. and Canada. In : Symbiotic nitrogen fixation in the management of temperate forests. Eds. Gordon J.C., Wheeler C.T., Perry D.A., Corvallis Univ., 403-419.

FISHER D.J., HAYEs A.L., 1981. Effects of some fungicides used against cereal pathogens on the growth of Rhizobium trifolii and its capacity to fix nitrogen in white clover. Ann. Appl. Biol., 98, 101-107.

FishrR D.J., Hayes A.L., 1982. Effects of some systemic imidazole and triazole fungicides on white clover and symbiotic nitrogen fixation by Rhizobium trifolii. Ann. Appl. Biol., 101, 19-24.

Fisher D.J., Hayes A.L., Jones C.A., 1978. Effects of some surfactant fungicides on Rhizobium trifolii and its symbiotic relationship with white clover. Ann. Appl. Biol., 90, 73-84.

Fisher D.J., Pickard J.A., Mckenzie C.M., 1979. Uptake of the systemic fungicide Triadimefon by clover and its effects on symbiotic nitrogen fixation. Pestic. Sci., 10, $75-82$.

Giardini A., Lopes E., Deuber R., 1978. Influence of herbicides on the nodulation of soybeans. In : Limitation and Potentials for biological nitrogen fixation in the tropics. Eds. Dobereiner J., Burcis R., Hollaender A., Plenum Press, 337 p. 
GiBson A.H., 1977. The influence of the environmental and managerial practics on the legume-Rhizobium symbiosis. In : A treatise on Dinitrogen fixation. Eds. Hardy R.W.F., Gibson A.H., Wiley and Sons Inc. Publ., Section IV, 393-450.

Kellison R.C., White G., 1979. Black alder performance in the Southeast. In : Symbiotic nitrogen fixation in the management of temperate forests. Eds. GORDON J.C., WHEELER C.T., Perry D.A., Corvallis Univ., 345-355.

Lalonde M., Calver H., Pine S., 1981. Isolation and use of Frankia strains in actinorhizae formation. In : Current perspectives in nitrogen fixation. Eds. Gibson A., NEwTON W., Australian Academy Sciences Canberra, 296-299.

Maynard C.A., Hall R.B., 1980. Early results of a range-wide provenance trial of Alnus glutinosa (L) Gaertn. Commun. 27th Northeast Forest Tree Improvement Conference. Burlington. Vermont U.S.A., 18 p.

Morroud A., Capellano A., 1978. Etude de la dynamique de l'azote à haute altitude : fixation d'azote (réduction de $\mathrm{C}_{2} \mathrm{H}_{2}$ ) par Alnus viridis Chaix et étude ultrastructurale des nodules. In : Symp. Physiologie des Racines et Symbioses. Nancy. Eds. RiEDACKer A., Gagnarie-Richard J., 365-371.

Molroud A., Caphli.ano A., 1979. Etude de la dynamique de l'azote à haute altitude. I : Fixation d'azote (réduction de $\mathrm{C}_{2} \mathrm{H}_{3}$ ) par Alnus viridis Chaix. Can. J. Bot., 57, $1979-1985$.

Moiroud A., Faure-Raynaud M., 1983. Influence de quelques herbicides à large spectre sur la croissance et l'infectivité de cultures pures de Frankia. Plant and Soil, 79, 133-136.

Normand P., Lalonde M., 1982. Evaluation of Frankia strains isolated from provenance of two Alnus species. Can. J. Microbiol., 28, 1133-1142.

PLASS W.T., 1977. Growth and survival of hardwood and pine interplanted with european alder. U.S.D.A. For. Serv, Res. paper N.E. 376, Upper Darby, Pcnn., 10 p.

SAUCIER J.R., 1977. Growth and survival of European black alder cnd sycamore on uplant and bottomland sites. U.S.D.A. For. Serv. Res. Note S.E. 248, 6 p.

SILVESTER W., 1977. Dinitrogen fixation by plant associations excluding legumes. In : $\boldsymbol{A}$ treatise on Dinitrogen fixation. Section IV. Eds. Hardy R.W.F., Gibson A.H., Wiley and Sons Inc. Publ., 141-190.

Torrey J., 1976. Initiation and development of root nodules of Casuarina (Casuarinaceae). Am. J. Bot., 63, 335-344.

VIERECK L.A., 1966. Plant succession and soil development on gravel outwash of the Muldrow glacier: Alaska. Ecol. Monogr., 136, 181-194.

VINCENT J.M., 1977. Rhizobium, general microbiology. In : A treatise on Dinitrogen fixation. Scction III. Eds. Hardy R.W.F., Silver W.S., WileY and Sons, New York, 277-366. 